\title{
Spectral projections for the twisted Laplacian
}

\author{
by \\ Herbert Koch (Dortmund) and Fulvio Ricci (Pisa)
}

\begin{abstract}
Let $n \geq 1, d=2 n$, and let $(x, y) \in \mathbb{R}^{n} \times \mathbb{R}^{n}$ be a generic point in $\mathbb{R}^{2 n}$. The twisted Laplacian$$
L=-\frac{1}{2} \sum_{j=1}^{n}\left[\left(\partial_{x_{j}}+i y_{j}\right)^{2}+\left(\partial_{y_{j}}-i x_{j}\right)^{2}\right]
$$

has the spectrum $\left\{n+2 k=\lambda^{2}: k\right.$ a nonnegative integer $\}$. Let $P_{\lambda}$ be the spectral projection onto the (infinite-dimensional) eigenspace. We find the optimal exponent $\varrho(p)$ in the estimate

$$
\left\|P_{\lambda} u\right\|_{L^{p}\left(\mathbb{R}^{d}\right)} \lesssim \lambda^{\varrho(p)}\|u\|_{L^{2}\left(\mathbb{R}^{d}\right)}
$$
\end{abstract}

for all $p \in[2, \infty]$, improving previous partial results by Ratnakumar, Rawat and Thangavelu, and by Stempak and Zienkiewicz. The expression for $\varrho(p)$ is

$$
\varrho(p)= \begin{cases}1 / p-1 / 2 & \text { if } 2 \leq p \leq 2(d+1) /(d-1) \\ (d-2) / 2-d / p & \text { if } 2(d+1) /(d-1) \leq p \leq \infty\end{cases}
$$

1. Introduction. Sharp $L^{2}-L^{p}$-bounds for spectral projections onto eigenspaces of elliptic differential operators $L$ with a discrete spectrum have attracted considerable attention in the last 20 years, starting with the work of C. Sogge [5] on the spherical Laplacian (see also [4]). If $P_{\lambda}$ is the spectral projection corresponding to an eigenvalue $\lambda^{2}$ of $L$ and $2 \leq p \leq \infty$, one looks for the best possible exponent $\varrho(p)$ such that

$$
\left\|P_{\lambda} u\right\|_{p} \lesssim \lambda^{\varrho(p)}\|u\|_{2}
$$

In general, $\varrho(p)$ is a convex function of $1 / p$. Strict convexity of $\varrho(p)$ at some points is connected with dispersive estimates for $L$ and in some cases to phenomena in harmonic analysis such as restriction theorems for the Fourier transform.

2000 Mathematics Subject Classification: 43A85, 35P20.

Key words and phrases: spectral projection, twisted Laplacian. 
We consider here the so-called twisted Laplacian

$$
L=-\frac{1}{2} \sum_{j=1}^{n}\left[\left(\partial_{x_{j}}+i y_{j}\right)^{2}+\left(\partial_{y_{j}}-i x_{j}\right)^{2}\right]
$$

in $\mathbb{R}^{2 n}$. In dimension 2 (i.e. $n=1$ ), $L$ can be viewed as a Schrödinger operator with the magnetic potential $A=(y,-x)$, inducing a constant magnetic field.

The sharp estimates (1) relative to $L$ are as follows.

Theorem 1. With $d=2 n$ and $2 \leq p \leq \infty$, (1) holds with

$$
\varrho(p)=\left\{\begin{array}{lc}
\frac{1}{p}-\frac{1}{2} & \text { if } 2 \leq p \leq \frac{2(d+1)}{d-1}, \\
\frac{d-2}{2}-\frac{d}{p} & \text { if } \frac{2(d+1)}{d-1} \leq p \leq \infty,
\end{array}\right.
$$

and with no smaller exponent.

The first partial result in this direction is in [3], where the second exponent in $(2)$ was obtained for $p$ larger than some $p_{0}>2(d+1) /(d-1)$. Later, Stempak and Zienkiewicz [6] obtained (2) for $p \neq 2(d+1) /(d-1)$.

Our approach is inspired by the recent work of Koch and Tataru [1] on dispersive estimates and their application to the Hermite operator [2]. In particular it is based on PDE techniques and it does not need the refined estimates on Laguerre functions, which are used in [6].

The basic estimate is the local dispersive estimate (8) below, which implies the endpoint result for $p=2(d+1) /(d-1)$ by a simple covering argument. Weaker local estimates were also used by Thangavelu. The other endpoint result at $p=\infty$ follows from the exact computation of the $L^{2}-L^{\infty}$ norm of $P_{\lambda}$ (see (6) below).

The connection between $L$ and Hermite operators is two-fold. On one hand, if we decompose $L^{2}\left(\mathbb{R}^{2 n}\right)$ as the orthogonal sum of the subspaces $V_{m}$ consisting of the functions $f$ such that $f\left(e^{i \theta_{1}}\left(x_{1}+i y_{1}\right), \ldots, e^{i \theta_{n}}\left(x_{n}+i y_{n}\right)\right)=e^{i\left(m_{1} \theta_{1}+\cdots+m_{n} \theta_{n}\right)} f\left(x_{1}+i y_{1}, \ldots, x_{n}+i y_{n}\right)$ $\left(m \in \mathbb{Z}^{n}\right)$, we see that

$$
L=-\frac{1}{2}\left(\Delta_{\mathbb{R}^{2 n}}-|x|^{2}-|y|^{2}\right)+i \sum_{j=1}^{n}\left(x_{j} \partial_{y_{j}}-y_{j} \partial_{x_{j}}\right)
$$

equals $-\frac{1}{2}\left(\Delta_{\mathbb{R}^{2 n}}-|x|^{2}-|y|^{2}\right)-\sum m_{j}$ on $V_{m}$.

On the other hand, the operators $X_{j}=\partial_{x_{j}}+i y_{j}$ and $Y_{j}=\partial_{y_{j}}-i x_{j}$ satisfy the canonical commutation relations $\left[X_{j}, Y_{k}\right]=-2 i \delta_{j, k}$. This implies that there is a unitary projective representation $\pi$ of $\mathbb{R}^{2 n}$ on $L^{2}\left(\mathbb{R}^{n}\right)$ (with variable $\xi)$, called the Weyl representation, such that $d \pi\left(X_{j}\right)=\sqrt{2} \partial_{\xi_{j}}$, $d \pi\left(Y_{j}\right)=-i \sqrt{2} \xi_{j}$. Then $d \pi(L)=-\Delta_{\mathbb{R}^{n}}+|\xi|^{2}$. The Stone-von Neumann 
theorem establishes an isomorphism between $L^{2}\left(\mathbb{R}^{2 n}\right)$ and $L^{2}\left(\mathbb{R}^{n}\right) \otimes L^{2}\left(\mathbb{R}^{n}\right)$, which intertwines the action of the twisted Laplacian on the first space with the action of the Hermite operator on the first factor in the tensor product.

Each of these arguments shows that $L$ has a discrete spectrum, equal to the set of integers $\lambda^{2}=n+2 k, k \in \mathbb{N}$, and that each eigenspace is infinite-dimensional. The precise description of the eigenspaces is given in Section 2.

The twisted Laplacian also describes the action of the Heisenberg subLaplacian on special classes of functions. On the reduced Heisenberg group $h_{n}=\mathbb{R}^{n} \times \mathbb{R}^{n} \times \mathbb{T}$ with product

$$
\left(x, y, e^{i \theta}\right)\left(x^{\prime}, y^{\prime}, e^{i \theta^{\prime}}\right)=\left(x+x^{\prime}, y+y^{\prime}, e^{i\left(\theta+\theta^{\prime}+x^{\prime} \cdot y-x \cdot y^{\prime}\right)}\right),
$$

consider the left-invariant sub-Laplacian

$$
\mathcal{L}=-\frac{1}{2} \sum_{j=1}^{n}\left[\left(\partial_{x_{j}}+y_{j} \partial_{\theta}\right)^{2}+\left(\partial_{y_{j}}-x_{j} \partial_{\theta}\right)^{2}\right] .
$$

If $f\left(x, y, e^{i \theta}\right)=g(x, y) e^{i m \theta}$ with $m \in \mathbb{Z}$, then

$$
\begin{aligned}
\mathcal{L} f\left(x, y, e^{i \theta}\right) & =-\frac{1}{2} e^{i m \theta} \sum_{j=1}^{n}\left[\left(\partial_{x_{j}}+i m y_{j}\right)^{2}+\left(\partial_{y_{j}}-i m x_{j}\right)^{2}\right] g(x, y) \\
& =e^{i m \theta} L_{m} g(x, y) .
\end{aligned}
$$

One easily verifies that, for $m \neq 0$, the spectrum of $L_{m}$ consists of the integers $|m|(n+2 k)$ with $k \in \mathbb{N}$, and that the pairs $(|m|(n+2 k), m)$ with $m \in \mathbb{Z} \backslash\{0\}, k \in \mathbb{N}$ give the discrete joint spectrum of $\mathcal{L}$ and $i^{-1} \partial_{\theta}$. If $P_{m, k}$ is the orthogonal projection on the joint eigenspace, a simple scaling argument shows that

$$
\left\|P_{m, k} u\right\|_{L^{p}\left(h_{n}\right)} \lesssim(n+2 k)^{\frac{1}{2} \varrho(p)}|m|^{\sigma(p)}\|u\|_{L^{2}\left(h_{n}\right)},
$$

with $\varrho(p)$ as in $(2)$ and $\sigma(p)=(d / 2)(1 / 2-1 / p)$.

Acknowledgments. This research was done during the Research Trimester on Harmonic Analysis at the Centro de Giorgi in Pisa. Both authors are members of the European IHP network HARP "Harmonic Analysis and Related Problems". We acknowledge the support for this work by the European Commission through the Network and the support by the Centro de Giorgi in Pisa.

\section{Spectrum, eigenfunctions and lower bounds for spectral pro-} jections. Here we introduce some notation, derive formulas for some eigenfunctions and calculate $L^{p}$-norms of them. This will imply that the bounds are sharp. 
We identify $\mathbb{R}^{n} \times \mathbb{R}^{n}$ with $\mathbb{C}^{n}$ by $z=x+i y$ and write

$$
\partial_{z_{j}}=\frac{1}{2}\left(\partial_{x_{j}}-i \partial_{y_{j}}\right), \quad \partial_{\bar{z}_{j}}=\frac{1}{2}\left(\partial_{x_{j}}+i \partial_{y_{j}}\right) .
$$

Then

$$
-\frac{1}{2}\left[\left(\partial_{x_{j}}+i y_{j}\right)^{2}+\left(\partial_{y_{j}}-i x_{j}\right)^{2}\right]=-\frac{1}{2}\left(2 \partial_{z_{j}}-\bar{z}_{j}\right)\left(2 \partial_{\bar{z}_{j}}+z_{j}\right)+1=\frac{1}{2} D_{j}^{*} D_{j}+1,
$$

with $D_{j}=2 \partial_{\bar{z}_{j}}+z_{j}$. Since

$$
D_{j}\left(f(z) e^{-|z|^{2} / 2}\right)=2 \partial_{\bar{z}_{j}} f(z) e^{-|z|^{2} / 2},
$$

for all holomorphic functions $f$,

$$
L\left[f(z) e^{-|z|^{2} / 2}\right]=n f(z) e^{-|z|^{2} / 2} .
$$

On the other hand,

$$
\int_{\mathbb{C}^{n}}(L u) \bar{u} d z=n\|u\|_{L^{2}}^{2}+\frac{1}{2} \sum_{j=1}^{n}\left\|D_{j} u\right\|_{L^{2}}^{2},
$$

which shows that $n$ is the smallest eigenvalue and that the corresponding eigenfunctions are annihilated by $D_{j}$. An easy calculation shows

$$
\left[D_{j}, D_{j}^{*}\right]=4
$$

and hence, if $f$ is an eigenfunction with eigenvalue $\mu$ then

$$
\begin{aligned}
L D_{j}^{*} f & =D_{j}^{*} L f+2 D_{j}^{*} f=(\mu+2) D_{j}^{*} f, \\
L D_{j} f & =D_{j} L f-2 D_{j} f=(\mu-2) D_{j} f .
\end{aligned}
$$

We obtain an orthogonal basis of eigenfunctions of the form

$$
f_{\alpha, \beta}(z)=\left(2^{-1} D^{*}\right)^{\alpha}\left(z^{\beta} e^{-|z|^{2} / 2}\right)=(-1)^{|\alpha|+|\beta|} e^{|z|^{2} / 2} \partial_{z}^{\alpha} \partial_{\bar{z}}^{\beta} e^{-|z|^{2}}
$$

with

$$
L f_{\alpha, \beta}=(n+2|\alpha|) f_{\alpha, \beta} .
$$

In particular,

$$
\bar{z}_{1}^{k} e^{-|z|^{2} / 2}=(-1)^{k} f_{(k, 0, \ldots), 0}(z)
$$

is an eigenfunction with eigenvalue $n+2 k$. When we consider the twisted Laplacian as a quantization of the motion of a charged particle in a constant magnetic field, then this eigenfunction corresponds to the motion in a circle of radius $\sqrt{k}$ around zero and its maximal concentration (to scale 1) around that circle is dictated by the uncertainty principle. Thus this eigenfunction behaves like the characteristic function of a neighborhood of size 1 around that circle. 
Its $L^{p}$-norm can be explicitly computed. Integrating in $z_{1}$ first, we obtain

$$
\begin{aligned}
\int_{\mathbb{C}}\left|z_{1}\right|^{k p} e^{-p\left|z_{1}\right|^{2} / 2} d z_{1} & =2 \pi \int_{0}^{\infty} r^{k p+1} e^{-p r^{2} / 2} d r=\frac{2 \pi}{p}\left(\frac{2}{p}\right)^{k p / 2} \int_{0}^{\infty} t^{k p / 2} e^{-t} d t \\
& =\frac{2 \pi}{p}\left(\frac{2}{p}\right)^{k p / 2} \Gamma\left(\frac{k p}{2}+1\right)
\end{aligned}
$$

and hence

$$
\left\|z_{1}^{k} e^{-|z|^{2} / 2}\right\|_{L^{p}\left(\mathbb{R}^{2 n}\right)}^{p}=\left(\frac{2 \pi}{p}\right)^{n}\left(\frac{2}{p}\right)^{k p / 2} \Gamma\left(\frac{k p}{2}+1\right) .
$$

By Stirling's formula

$$
\Gamma(t+1) \sim \sqrt{2 \pi t}(t / e)^{t}
$$

and so

$$
\left\|z_{1}^{k} e^{-|z|^{2} / 2}\right\|_{L^{p}\left(\mathbb{R}^{2 n}\right)} /\left\|z_{1}^{k} e^{-|z|^{2} / 2}\right\|_{L^{2}\left(\mathbb{R}^{2 n}\right)} \sim k^{(1 / p-1 / 2) / 2} .
$$

This proves that $\varrho(p) \geq 1 / p-1 / 2$.

We shall now need the $L^{2}$-norms of all the $f_{\alpha, \beta}$. We can reduce ourselves to one dimension, since

$$
f_{\alpha, \beta}(z)=\prod_{j=1}^{n} f_{\alpha_{j}, \beta_{j}}\left(z_{j}\right) .
$$

In dimension $n=1$, the computation in (4) shows that

$$
\left\|f_{0, \ell}\right\|_{2}^{2}=\pi \ell !
$$

Observe next that

$$
\begin{aligned}
\left\|f_{k, \ell}\right\|_{2}^{2} & =\frac{1}{4}\left\|D^{*} f_{k-1, \ell}\right\|_{2}^{2}=\frac{1}{4}\left\langle f_{k-1, \ell}, D D^{*} f_{k-1, \ell}\right\rangle \\
& =\frac{1}{4}\left\langle f_{k-1, \ell},\left(D^{*} D+\left[D, D^{*}\right]\right) f_{k-1, \ell}\right\rangle \\
& =\frac{1}{4}\left\langle f_{k-1, \ell},(2 L+2) f_{k-1, \ell}\right\rangle=k\left\|f_{k-1, \ell}\right\|_{2}^{2},
\end{aligned}
$$

so that $\left\|f_{k, \ell}\right\|_{2}^{2}=\pi k ! \ell$, and, in $n$ dimensions,

$$
\left\|f_{\alpha, \beta}\right\|_{2}^{2}=\pi^{n} \alpha ! \beta ! \text {. }
$$

As for the Hermite operator [2], we expect that radial eigenfunctions will provide the sharp value of $\varrho(p)$ for $p$ close to $\infty$.

For $k \in \mathbb{N}$, consider

$$
\begin{aligned}
f_{k}(z) & =e^{|z|^{2} / 2} \Delta_{\mathbb{R}^{2 n}}^{k} e^{-|z|^{2}}=4^{-k} e^{|z|^{2} / 2}\left(\sum_{j=1}^{n} \partial_{z_{j}} \partial_{\bar{z}_{j}}\right)^{k} e^{-|z|^{2}} \\
& =4^{-k} \sum_{|\alpha|=k}\left(\begin{array}{c}
k \\
\alpha
\end{array}\right) f_{\alpha, \alpha}(z) .
\end{aligned}
$$


Then $f_{k}$ is radial, and it is an eigenfunction of $L$ with eigenvalue $n+2 k$. Since for radial functions the twisted Laplacian and the Hermite operator coincide up to a factor 2 , the $f_{k}$ are the unique (up to scalar multiples) radial eigenfunctions of $L$.

By (5) and the orthogonality of the $f_{\alpha, \alpha}$,

$$
\left\|f_{k}\right\|_{2}^{2}=4^{-2 k} \pi^{n} \sum_{|\alpha|=k} k !^{2}=4^{-2 k} \pi^{n} k !^{2}\left(\begin{array}{c}
n+k-1 \\
k
\end{array}\right) .
$$

From (3) we see that $f_{\alpha, \alpha}(0)=\alpha$ !, hence

$$
f_{k}(0)=4^{-k} k !\left(\begin{array}{c}
n+k-1 \\
k
\end{array}\right) \text {. }
$$

Thus

$$
\frac{\left\|f_{k}\right\|_{L^{\infty}}}{\left\|f_{k}\right\|_{L^{2}}} \geq \frac{f_{k}(0)}{\left\|f_{k}\right\|_{L^{2}}}=\pi^{-n / 2} \sqrt{\left(\begin{array}{c}
n+k-1 \\
k
\end{array}\right)} \sim k^{(d-2) / 4} .
$$

This proves that $\varrho(\infty) \geq(d-2) / 2$.

It is not hard to see that the first inequality is in fact an equality, and that the ratio $\left\|f_{k}\right\|_{L^{\infty}} /\left\|f_{k}\right\|_{L^{2}}$ coincides with the $L^{2}-L^{\infty}$-norm of the spectral projection. If $f$ is an eigenfunction with eigenvalue $n+2 k$ of the twisted Laplacian with $L^{2}$ norm 1 which maximizes the $L^{\infty}$ norm then we may assume, after a twisted translation of the form $f(x, y) \mapsto e^{i(a \cdot y-b \cdot x)} f(x-$ $a, y-b)$, that it assumes its maximum at $z=0$. Averaging over the unitary group $U(n)$ we see that it has to be radial, hence a scalar multiple of $f_{k}$. Thus, for $\lambda^{2}=n+2 k$,

$$
\left\|P_{\lambda}\right\|_{2 \rightarrow \infty}=\pi^{-n} \sqrt{\left(\begin{array}{c}
n+k-1 \\
k
\end{array}\right)} .
$$

We now look for an estimate from below of $\left\|f_{k}\right\|_{p}$ for $p$ finite. Since $f_{k}$ is radial we obtain by the divergence theorem, if $|z|=r$,

$$
\begin{aligned}
\partial_{r} f_{k}(z) & =\frac{c_{d}}{r^{d-1}} \int_{S_{r}} \partial_{\nu} f_{k}(w) d \sigma(w)=\frac{c_{d}}{r^{d-1}} \int_{B_{r}} \Delta f_{k}(w) d w \\
& =\frac{c_{d}}{r^{d-1}} \int_{B_{r}}\left(-2 L+|w|^{2}\right) f_{k}(w) d w \\
& =\frac{c_{d}}{r^{d-1}} \int_{B_{r}}\left(-2 n-4 k+|w|^{2}\right) f_{k}(w) d w
\end{aligned}
$$

Thus, for $r<1$,

$$
\left|\partial_{r} f_{k}(z)\right| \leq C r(n+2 k)\left\|f_{k}\right\|_{\infty}=C r(n+2 k) f_{k}(0),
$$


and

$$
f_{k}(z) \geq f_{k}(0)\left(1-C(n+2 k)|z|^{2}\right) .
$$

It follows that for $|z|<r_{k}=1 / \sqrt{C(n+2 k)}, f_{k}(z)>\frac{1}{2} f_{k}(0)$, so that

$$
\left\|f_{k}\right\|_{p} \geq C r_{k}^{d / p} f_{k}(0) \sim k^{-d / 2 p+(d-2) / 4}\left\|f_{k}\right\|_{2} .
$$

This shows that $\varrho(p) \geq(d-2) / 2-d / p$.

3. Upper bounds for spectral projections. Let $\lambda^{2}=n+2 k$ and

$$
L u=\lambda^{2} u \text {. }
$$

Because of (6) the assertion follows once we prove for all $\left(x_{0}, y_{0}\right)$ that

$$
\lambda^{1 /(d+1)}\|u\|_{L^{2(d+1) /(d-1)}\left(B_{\lambda}\left(x_{0}, y_{0}\right)\right)} \lesssim\|u\|_{L^{2}\left(B_{2 \lambda}\left(x_{0}, y_{0}\right)\right)} .
$$

We set

$$
x=x_{0}+\lambda \bar{x}, \quad y=y_{0}+\lambda \bar{y} \quad \bar{u}(\bar{x}, \bar{y})=e^{-i\left(x_{0} y-y_{0} x\right)} u(x, y) .
$$

Then

$$
\left(\lambda^{-1} \partial_{\bar{x}_{j}}+i \lambda \bar{y}_{j}\right) \bar{u}=\left(\partial_{x_{j}}+i\left(y_{j}-\left(y_{0}\right)_{j}\right) e^{-i\left(x_{0} y-y_{0} x\right)} u=e^{-i\left(x_{0} y-y_{0} x\right)}\left(\partial_{x_{j}}+i y_{j}\right) u\right.
$$

and hence

$$
\bar{L} \bar{u}:=-\frac{1}{2} \sum_{j=1}^{n}\left(\left(\partial_{\bar{x}_{j}}+i \lambda^{2} \bar{y}_{j}\right)^{2}+\left(\partial_{\bar{y}_{j}}-i \lambda^{2} \bar{x}_{j}\right)^{2}\right) \bar{u}=\lambda^{4} \bar{u} .
$$

We drop the bar on $x, y$ and $u$ but not on $L$. Hence we study

$$
\bar{L} u=\lambda^{4} u
$$

in a ball of radius 2 . The inequality (7) takes the form

$$
\lambda^{2 /(d+1)}\|u\|_{L^{2(d+1) /(d-1)}\left(B_{1}(0)\right)} \lesssim \lambda\|u\|_{L^{2}\left(B_{2}(0)\right)} .
$$

Actually a slightly stronger bound is true:

Lemma 2. Suppose that

$$
L_{\mu} u:=-\frac{1}{2} \sum_{j=1}^{n}\left(\left(\partial_{\bar{x}_{j}}+i \mu \bar{y}_{j}\right)^{2}+\left(\partial_{\bar{y}_{j}}-i \mu \bar{x}_{j}\right)^{2}\right) u-\mu^{2} u=f .
$$

Then

$$
\mu^{1 /(d+1)}\|u\|_{L^{2(d+1) /(d-1)}\left(B_{1}(0)\right)} \lesssim \mu^{1 / 2}\|u\|_{L^{2}\left(B_{2}(0)\right)}+\mu^{-1 / 2}\|f\|_{L^{2}\left(B_{2}(0)\right)} .
$$

The dispersive estimate (8) is an immediate consequence.

Proof. The statement of Lemma 2 follows from Theorem 2 of [1] in the same way as Lemma 3.4 of [2] is deduced from the same result. The symbol of $L_{\mu}$ is

$$
p(x, y, \xi, \eta)=\frac{1}{2}\left(|\xi+y|^{2}+|\eta-x|^{2}\right)-\mu^{2},
$$


which is real. Keeping $x$ and $y$ fixed it vanishes on a sphere of radius $\mu$, which has $2 n-1$ nonvanishing curvatures of size $\mu^{-1}$. This curvature leads to the desired estimate.

\section{References}

[1] H. Koch and D. Tataru, Dispersive estimates for principally normal operators, Comm. Pure Appl. Math. 58 (2005), 217-284.

[2] - - - $L^{p}$ eigenfunction bounds for the Hermite operator, Duke Math. J. 28 (2005), 369-382.

[3] P. K. Ratnakumar, R. Rawat, and S. Thangavelu, A restriction theorem for the Heisenberg motion group, Studia Math., 126 (1997), 1-12.

[4] C. D. Sogge, Concerning the $L^{p}$ norm of spectral clusters for second-order elliptic operators on compact manifolds, J. Funct. Anal. 77 (1988), 123-138.

[5] - Fourier Integrals in Classical Analysis, Cambridge Tracts in Math. 105, Cambridge Univ. Press, Cambridge, 1993.

[6] K. Stempak and J. Zienkiewicz, Twisted convolution and Riesz means, J. Anal. Math. 76 (1998), 93-107.

Mathematisches Institut

Universität Bonn

Beringstr. 1

53115 Bonn, Germany

E-mail: koch@math.uni-bonn.de
Scuola Normale Superiore

Piazza dei Cavalieri 7 56126 Pisa, Italy E-mail: fricci@sns.it

Received December 13, 2004

Revised version April 13, 2005 